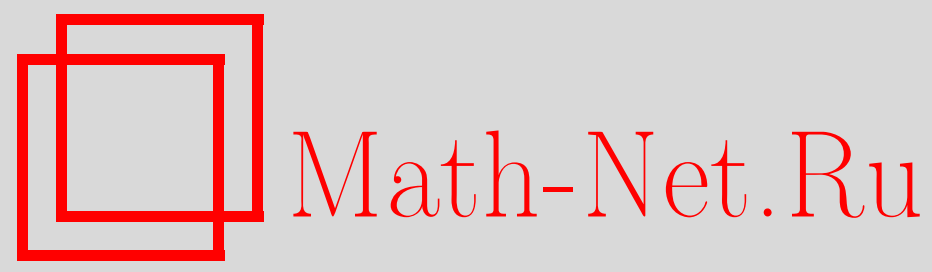

А.-Р. К. Рамазанов, Оценка нормы полианалитической функции через норму ее полигармонической составляющей, Матем. заметки, 2004, том 75, выпуск 4, 608-613

DOI: https://doi.org/10.4213/mzm56

Использование Общероссийского математического портала Math-Net.Ru подразумевает, что вы прочитали и согласны с пользовательским соглашением http://www.mathnet.ru/rus/agreement

Параметры загрузки:

IP : 3.85 .5 .30

26 апреля 2023 г., 04:31:54 


\section{ОЦЕНКА НОРМЫ ПОЛИАНАЛИТИЧЕСКОЙ ФУНКЦИИ ЧЕРЕЗ НОРМУ ЕЕ ПОЛИГАРМОНИЧЕСКОЙ СОСТАВЛЯЮЩЕЙ}

\section{А. К. Рамазанов}

Получена оценка для нормы полианалитической функции из пространства $L_{p}(D, \alpha)$, $1 \leqslant p<\infty, \alpha>-1$, через норму ее полигармонической составляющей.

Библиография: 15 названий.

Функция $f(z)$, которая имеет в области $G$ непрерывные частные производные по $x$ и $y$ до порядка $m \geqslant 1$, назьвается полианалитической функиией (n.a.ф.) порядка $m$ в области $G$, если в этой области она удовлетворяет обобшенному уравнению Коши-Римана $\partial^{m} f / \partial \bar{z}^{m}=0$.

Известно, что любую п.а.ф. порядка $m$ в области $G$ можно единственным образом представить в виде (см. [1])

$$
f(z)=\varphi_{0}(z)+\bar{z} \varphi_{1}(z)+\cdots+\bar{z}^{m-1} \varphi_{m-1}(z)
$$

где $\varphi_{k}$ голоморфны в $G$.

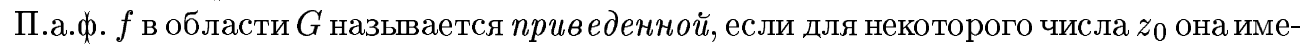
ет представление

$$
f(z)=\sum_{k=0}^{m-1} h_{k}(z)\left|z-z_{0}\right|^{2 k}
$$

где $h_{k}$ голоморфны в $G$.

Очевидно, что если $f$ - п.а.ф. порядка $m$ в области $G$, то $z^{m-1} f(z)$ является приведенной п.а.ф. порядка $m$ в области $G$.

Функции $\varphi_{k}$ и $h_{k}$, будем называть голоморфными компонентами п.а.ф.

Функция $u(x, y)$, которая является решением уравнения

$$
\Delta^{m} u(x, y)=0
$$

в области $G$, называется полигармонической функиией порядка $m$ в области $G$. Известно, что если $f$ - п.а.ф. порядка $m$ в области $G$, то ее действительная и мнимая части являются полигармоническими функциями порядка не выше $m$ в области $G$ (см., например, [1]).

Работа поддержана Российским фондом фундаментальных исследований, грант № 02-01-00913, РФФИ-БРФФИ, грант № 02-01-81031, и Государственной программой поддержки ведущих научных школл, грант № НШ-1892.2003.1. 
Пусть $D=\{z:|z|<1\}$ и $L_{p}(D, \alpha), 1 \leqslant p \leqslant \infty, \alpha>-1$, обозначает пространство функций $f$, для которых конечна норма

$$
\|f\|_{(p, D, \alpha)}=\left\{\iint_{D}\left(1-|z|^{2}\right)^{\alpha}|f(z)|^{p} d x d y\right\}^{1 / p}
$$

Далее, пусть $A_{k}(D)$ - множество всех $k$-аналитических в $D$ функций $\left(A_{1}(D)=A(D)\right)$; $A_{k} L_{p}(D, \alpha)=A_{k}(D) \cap L_{p}(D, \alpha)$;

$$
\begin{aligned}
A_{k} L_{p}^{0}(D, \alpha)=\{ & f(z)=\left(1-|z|^{2}\right)^{-\alpha} \frac{\partial^{k-1}}{\partial z^{k-1}}\left((1-z \bar{z})^{k-1+\alpha} F(z)\right): F \in A(D), \\
& \left.f \in A_{k} L_{p}(D, \alpha)\right\} .
\end{aligned}
$$

Очевидно, что $A_{1} L_{p}(D, \alpha)=A L_{p}(D, \alpha)=A_{1} L_{p}^{0}(D, \alpha)$. Пространства $A_{k} L_{p}^{0}(D, \alpha)$ введены и исследованы в работах [2]-[4]. Отметим также работы [5], [6], где введены различные классы полианалитических функций для исследования граничного поведения компонент п.а.ф.

Известно, что норму аналитической функции $f(z)$ из $L_{p}(D, \alpha), 0<p<\infty$, можно оценить через норму ее действительной части $u(z)(u(0)=f(0))$ :

$$
\|f\|_{(p, D, \alpha)} \leqslant C(p, \alpha)\|u\|_{(p, D, \alpha)}
$$

где величина $C(p, \alpha)$ зависит лишш от указанных в скобках параметров.

При $1<p<\infty$ это неравенство является следствием известного неравенства Рисса об оценке нормы сопряженной гармонической функции $v(z)$ через норму самой гармонической функции $u(z)$ в пространстве Харди $H_{p}(D), 1<p<\infty$. Для $p=1$ оценка (3) получена в работе [7], а при $0<p \leqslant 1$ она доказана в [8]. Отметим, что при $p=\infty$ неравенство (3) не выполняется. Изучению оценок вида (3) для областей в комплексной плоскости посвящена обширная литература. Укажем лишш работы [9] и [10], где эта задача рассматривается с историей вопроса. В данной работе мы рассматриваем аналогичную задачу для п.а.ф.

ТЕОрема 1. Пусть $u(z)=u(x, y)$ - полигармоническая функиия порядка $m$ в односвязной области $G, 0 \in G$. Тогда существует единственная приведенная полианалитическая функиия порядка $m$ в области $G$

$$
f(z)=\sum_{k=0}^{m-1} h_{k}(z)|z|^{2 k}
$$

которая удовлетворяет следующим условиям:

1) $u(x, y)=\operatorname{Re}\{f(z)\}$

2) $u(0)=f(0)=h_{0}(0)$;

3) $\left.\Delta^{k} u(x, y)\right|_{z=0}=4^{k}(k !)^{2} h_{k}(0), \quad k=1, \ldots, m-1$. 
ДоКАЗАТЕЛЬСТво теоремы 1 , без условий 2) и 3), имеется в [1, с. 12]. Условия 2) и 3 ) получаются непосредственно, повторяя рассуждения доказательства, приведенного в [1].

ТЕОРема 2. Пусть полигармоническая функиия $и(x, y)$ порядка т принадлежит пространству $L_{p}(D, \alpha), 1 \leqslant p<\infty, u f(z)=\sum_{k=0}^{m-1} h_{k}(z)|z|^{2 k}-$ приведенная п.а.ф. порядка $m$, которая удовлетворяет условиям теоремы 1. Тогда

$$
\|f\|_{(p, D, \alpha)} \leqslant C(p, m, \alpha)\|u\|_{(p, D, \alpha)},
$$

где $C(p, D, \alpha)$ - величина, которая зависит лишь от указанных в скобках параметров.

ЗАмЕчАниЕ. Утверждение теоремы 2 при $\alpha=0$ и определенных ограничениях на п.а.ф. $f$ анонсировано в [11], а при любых $\alpha>-1-$ в [12].

Доказательство теоремы 2 опирается на ряд вспомогательных утверждений. Сначала введем обозначения: Г $(a)$ - гамма функция Эйлера,

$$
\begin{aligned}
& \mathscr{R}_{k}^{0, \alpha}(z, \zeta)=\frac{1}{\pi(k-1) !} \frac{\Gamma(\alpha+1)}{\Gamma(k+\alpha)}\left(1-|z|^{2}\right)^{-\alpha}\left(1-|\zeta|^{2}\right)^{-\alpha} \\
& \times \frac{\partial^{2(k-1)}}{\partial z^{(k-1)} \partial \bar{\zeta}^{(k-1)}}\left\{(1-z \bar{z})^{k-1+\alpha}(1-\zeta \bar{\zeta})^{k-1+\alpha} \frac{(k-1)(1-z \bar{\zeta})+\alpha+1}{(1-z \bar{\zeta})^{\alpha+2}}\right\}, \\
& \mathscr{R}_{m}^{\alpha}(z, \zeta)=\sum_{k=1}^{m} \mathscr{R}_{k}^{0, \alpha}(z, \zeta), \quad z, \zeta \in D, \quad k=1, \ldots, m .
\end{aligned}
$$

Очевидно, что $\mathscr{R}_{k}^{0, \alpha}(z, \zeta)=\overline{\mathscr{R}_{k}^{0, \alpha}(\zeta, z)}$ и при фиксированном $\zeta \in D$ п.а.ф. $\mathscr{R}_{k}^{0, \alpha}(z, \zeta)$ принадлежит пространству $A_{k} L_{p}^{0}(D, \alpha)$. Ядра $\mathscr{R}_{k}^{0, \alpha}(z, \zeta)$ введены в работах [2]-[4].

Введем следующий интегральньй оператор

$$
P_{k}^{0, \alpha}(f)(\zeta)=\iint_{D} f(z) \overline{\mathscr{R}}_{k}^{0, \alpha}(z, \zeta) d x d y, \quad f \in L_{p}(D, \alpha), \quad \zeta \in D, \quad z=x+i y \in D
$$

Оператор, получаемый из формулы (4) при замене ядра $\mathscr{R}_{k}^{0, \alpha}(z, \zeta)$ на ядро $\mathscr{R}_{m}^{\alpha}(z, \zeta)$, будем обозначать через $P_{m}^{\alpha}$. Очевидно, что $P_{m}^{\alpha}=\sum_{k=1}^{m} P_{k}^{0, \alpha}$. Операторы $P_{m}^{\alpha}$ исследованы в работах различных авторов (см. [2]-[4], [13], [14]).

При доказательстве некоторых вспомогательных утверждений также будем использовать ортогональность следующей системы функций в пространстве $L_{2}(D, \alpha)$ (см. [2] и [3]):

$$
e_{k, n}(z)=\frac{1}{\sqrt{\pi}} \sqrt{\frac{\Gamma(n+\alpha+1)(k+n+\alpha)}{\Gamma(k) \Gamma(k+\alpha) \Gamma(n+1)}}\left(1-|z|^{2}\right)^{-\alpha} \frac{\partial^{k-1}}{\partial z^{k-1}}\left\{(1-z \bar{z})^{k-1+\alpha} z^{n}\right\}
$$

где $k$ - любое натуральное число, а $n=0,1, \ldots$.

Лемма 1. 1) Oператор $P_{k}^{0, \alpha}$ (соответственно $P_{m}^{\alpha}$ ) является оператором проектирования пространства $L_{p}(D, \alpha)$ на его подпространство $A_{k} L_{p}^{0}(D, \alpha)$ (соответственно $\left.A_{m} L_{p}(D, \alpha)\right)$ при $1<p<\infty$ и всех $\alpha>-1$.

2) Если $f \in A_{k} L_{p}^{0}(D, \alpha)$ (соответственно $f \in A_{m} L_{p}(D, \alpha)$ ), mо $P_{k}^{0, \alpha}(f)(z)=f(z)$, $z \in D$ (соответственно $\left.P_{m}^{\alpha}(f)(z)=f(z), z \in D\right)$ при всех $1 \leqslant p \leqslant \infty$.

Доказательство леммы 1 имеется в [4] (см. теорему 2). 
ЛЕмма 2. Пусть приведенная п.а.ф. $f(z)=\sum_{j=0}^{m-1} h_{j}(z)|z|^{2 j}$ порядка т принадлежит пространству $A_{m} L_{p}(D, \alpha), 1 \leqslant p<\infty, u h_{j}(z)=\sum_{\nu=0}^{\infty} a_{j, \nu} z^{\nu}$. Тогда

$$
\mathscr{P}_{k}^{0, \alpha}(\bar{f})(z)=\left(1-|z|^{2}\right)^{-\alpha}(-1)^{k-1} \sum_{n=0}^{k-1} c_{k, n} \frac{\partial^{k-1}}{\partial z^{k-1}}\left((1-z \bar{z})^{k-1+\alpha} z^{n}\right)
$$

$2 \partial e$

$$
c_{k, n}=\frac{\Gamma(n+\alpha+1)(k+n+\alpha)}{\Gamma(k) \Gamma(n+1)} \sum_{j=n}^{m-1} \bar{a}_{j, k-1-n} \frac{\Gamma(k-n+j) \Gamma(j+1)}{\Gamma(j-n+1) \Gamma(k+j+\alpha+1)}
$$

nрu $k=1,2, \ldots, m$.

ДокАЗАТЕЛЬство леммы 2 проводим по следующей схеме. Сначала равенство (5) доказьваем для приведенного полимногочлена порядка $m$ с использованием свойства ортогональности функций $e_{k, n}(z), k=1,2, \ldots, m, n=0,1, \ldots$ После этого используем предельньй переход.

ЛЕмма 3. Пусть приведенная п.а.ф. $f(z)=\sum_{j=0}^{m-1} h_{j}(z)|z|^{2 j}$ порядка т принадлехит пространству $A_{m} L_{p}(D, \alpha), 1 \leqslant p<\infty, u h_{j}(z)=\sum_{\nu=0}^{\infty} a_{j, \nu} z^{\nu}$. Тогда для $u(z)=\operatorname{Re}\{f(z)\}$ имеем

$$
\iint_{D}\left(1-|z|^{2}\right)^{\alpha} u(z) \overline{e_{k, n}(z)} d x d y=\frac{(-1)^{k-1}}{2} \sqrt{\pi} \sqrt{\frac{\Gamma(n+\alpha+1)(k+n+\alpha)}{\Gamma(k) \Gamma(k+\alpha) \Gamma(n+1)}} d(n, k, m)
$$

əəe

$$
d(n, k, m)=\left\{\begin{array}{lc}
\sum_{j=n}^{m-1} \bar{a}_{j, k-1-n} \frac{\Gamma(k-n+j) \Gamma(j+1) \Gamma(k+\alpha)}{\Gamma(j-n+1) \Gamma(k+j+\alpha+1)} & \text { npu } n<k-1, \\
2 \sum_{j=k-1}^{m-1} a_{j, 0} \frac{(\Gamma(j+1))^{2} \Gamma(k+\alpha)}{\Gamma(j-(k-1)+1) \Gamma(k+j+\alpha+1)} & \text { npu } n=k-1, \\
\sum_{j=k-1}^{m-1} a_{j, n-(k-1)} \frac{\Gamma(j+1) \Gamma(k+\alpha) \Gamma(j-(k-1)+n+1)}{\Gamma(j-(k-1)+1) \Gamma(j+n+\alpha+2)} & n p u n>k-1 .
\end{array}\right.
$$

ДокАЗАТЕЛЬСТво леммы 3 аналогично доказательству леммы 2 .

Лемма 4. Для любых $z, \zeta \in D$ справедливо неравенство

$$
\left|\mathscr{R}_{m}^{\alpha}(z, \zeta)\right| \leqslant \frac{C(\alpha, m)}{|1-z \bar{\zeta}|^{2+\alpha}} .
$$

Доказательство леммы 4 имеется в работах [14] и [4]. 
Лемма 5. Пусть $\alpha>-1, \beta>0 u z, \zeta \in D$. Тогда

$$
\iint_{D} \frac{\left(1-|z|^{2}\right)^{\alpha}}{|1-z \bar{\zeta}|^{2+\alpha+\beta}} d x d y \leqslant C(\alpha, \beta)\left(1-|z|^{2}\right)^{-\beta}
$$

Доказательство леммы 6 приведено в [15].

ДоКАЗАТЕЛЬСТВО ТЕОРЕМЫ 2. По Пункту 2) леммы 1

$$
f(z)=\mathscr{P}_{m}^{\alpha}(f)(z)=\mathscr{P}_{m}^{\alpha}(f+\bar{f})(z)-\mathscr{P}_{m}^{\alpha}(\bar{f})(z)=2 \mathscr{P}_{m}^{\alpha}(u)(z)-\sum_{k=1}^{m} \mathscr{P}_{k}^{0, \alpha}(\bar{f})(z) .
$$

Из лемм 2 и 3 следует, что

$$
\left|\mathscr{P}_{m}^{\alpha}(\bar{f})(z)\right| \leqslant C_{1}(p, m, \alpha)\|u\|_{(p, D, \alpha)} .
$$

Тогда, используя ограниченность оператора $\mathscr{P}_{m}^{\alpha}$ в пространстве $L_{p}(D, \alpha), 1<p<\infty$, получаем

$$
\begin{aligned}
\|f\|_{(p, D, \alpha)} & \leqslant\left\|\mathscr{P}_{m}^{\alpha}(u)\right\|_{(p, D, \alpha)}+C_{2}(p, m, \alpha)\|u\|_{(p, D, \alpha)} \\
& \leqslant C_{3}(p, m, \alpha)\|u\|_{(p, D, \alpha)}+C_{2}(p, m, \alpha)\|u\|_{(p, D, \alpha)} \leqslant C(p, m, \alpha)\|u\|_{(p, D, \alpha)} .
\end{aligned}
$$

Рассмотрим теперь случай $p=1$. Очевидно, что $f \in A_{m} L_{1}\left(D, \alpha+\alpha_{0}\right)$ при любом $\alpha_{0}>0$. Пусть $\alpha_{0}=1$. Тогда, как и вьше, по лемме 1 имеем

$$
f(\zeta)=\mathscr{P}_{m}^{\alpha+1}(f)(\zeta)=2 \mathscr{P}_{m}^{\alpha+1}(u)(\zeta)-\mathscr{P}_{m}^{\alpha+1}(\bar{f})(\zeta)
$$

Применив леммы 2 и 3 еще раз, оцениваем второе слагаемое суммы (6):

$$
\left|\mathscr{P}_{m}^{\alpha+1}(\bar{f})(z)\right| \leqslant C_{1}(m, \alpha)\|u\|_{(1, D, \alpha+1)} \leqslant C_{1}(m, \alpha)\|u\|_{(1, D, \alpha)} .
$$

Обозначим через $d \sigma(z)$ меру Лебега на плоскости. Тогда из (6) получаем

$$
\begin{aligned}
\|f\|_{(1, D, \alpha)} \leqslant & 2 \iint_{D}\left(1-|z|^{2}\right)^{\alpha+1}|u(z)| \\
& \times\left(\iint_{D}\left(1-|\zeta|^{2}\right)^{\alpha}\left|\mathscr{R}_{m}^{\alpha+1}(z, \zeta)\right| d \sigma(\zeta)\right) d \sigma(z)+C_{1}(m, \alpha)\|u\|_{(1, D, \alpha)} .
\end{aligned}
$$

Применив к внутреннему интегралу лемму 4 , а затем лемму 5 , из последнего неравенства получаем

$$
\begin{aligned}
\|f\|_{(1, D, \alpha)} \leqslant & 2 C \iint_{D}\left(1-|z|^{2}\right)^{\alpha+1}|u(z)| \\
& \left.\times \iiint_{D}\left(1-|\zeta|^{2}\right)^{\alpha} \frac{1}{|1-z \bar{\zeta}|^{2+\alpha+1}} d \sigma(\zeta)\right) d \sigma(z)+C_{1}(m, \alpha)\|u\|_{(1, D, \alpha)} \\
\leqslant & 2 C \iint_{D}\left(1-|z|^{2}\right)^{\alpha+1}|u(z)| \frac{1}{\left(1-|z|^{2}\right)^{1}} d \sigma(z)+C_{1}(m, \alpha)\|u\|_{(1, D, \alpha)} \\
= & C(m, \alpha)\|u\|_{(1, D, \alpha)} .
\end{aligned}
$$

Таким образом, теорема 2 полностью доказана. 


\section{СПИСОК ЦИТИРОВАННОЙ ЛИТЕРАТУРЫ}

[1] Balk M. B. Polyanalytic Functions. Berlin: Akad. Verlag, 1991.

[2] Рамазанов А.К. Об ортогональных проекторах в пространствах полианалитических функций // Изв. ТулГУ. Сер. матем., мех., информатика. 1998. Т. 4. №1. С. 117-122.

[3] Рамазанов А.К. Представление пространства полианалитических функций в виде прямой суммы ортогональных подпространств. Приложение к рациональным аппроксимациям // Матем. заметки. 1999. Т. 66. № 5. С. 741-759.

[4] Рамазанов А. К. О строении пространств полианалитических функций // Матем. заметки. 2002. T. 72. № 5. C. 750-764.

[5] Долженко Е. П. О граничном поведении компонент полианалитической функции // Матем. заметки. 1998. Т. 63. №6. С. 821-834.

[6] Долженко Е. П., Данченко В. И. О граничном поведении решений обобщенного уравнения Коши-Римана // Вестн. МГУ. Сер. 1. Матем., мех. 1998. № 3. С. 16-25.

[7] Duren P. L., Shields A. L. Properties of $H^{p}(0<p<1)$ and its containing Banach space // Trans. Amer. Math. Soc. 1969. V. 141. P. 255-262.

[8] Шамоян Ф. А. Приложения интегральных представлений Джрбашяна к некоторым задачам анализа // Докл. АН СССР. 1981. Т. 261. № 3. С. 557-561.

[9] Burbea J. The Bergman projection over plane regions // Ark. Mat. 1980. V. 18. № 2. P. 207-221.

[10] Соловьев А. А. Оценки в $L^{p}$ интегральных операторов, связанных с пространствами аналитических и гармонических функций // Сиб. матем. ж. 1985. Т. 26. № 3. С. 168-191.

[11] Рамазанов А. К. Оценка нормы полианалитической функции через норму ее полигармонической составляющей // Современные методы теории функций и смежные проблемы. Тезисы докл. Воронеж: ВГУ, 1999. С. 163.

[12] Рамазанов А. К. Оценка нормы полианалитической функции через норму ее полигармонической составляющей // Современные проблемы теории функций и их приложения. Тезисы докл. 11-й Саратовской зимней школы. Саратов: СГУ, 2002. С. 166-167.

[13] Кошелев А. Д. О керн-функции для гильбертова пространства полианалитических функций в круге // Докл. АН СССР. 1977. Т. 232. № 2. С. 277-279.

[14] Васин А. В. Проекторы на $L_{p}$-пространства полианалитических функций // Записки научн. семин. ЛОМИ. 1991. Т. 190. С. 15-33.

[15] Рудин У. Теория функций в единичном шаре из $\mathbb{C}^{n}$. М.: Мир, 1984.

Калужский филиал Московского государственного

Поступило

технического университета им. Н. Э. Баумана

24.11.2002 